\title{
New Italian immunisation plan is built on scientific evidence: Carlo Signorelli and colleagues reply to news article by Michael Day
}

\author{
Carlo Signorelli professor of public health and president ${ }^{1}$, Anna Odone research student ${ }^{2}$, Paolo \\ Bonanni coordinator, working group on vaccines ${ }^{1}$, Francesca Russo director for prevention and \\ health, Veneto region ${ }^{3}$
}

${ }^{1}$ Italian Society of Hygiene, Preventive Medicine and Public Health (SItI), 00144 Rome, Italy; ${ }^{2}$ Unit of Public Health, Department of Biomedical, Biotechnological and Translational Sciences, University of Parma, 43125 Parma, Italy; ${ }^{3}$ Dorsoduro, 3493 Venezia, Italy

Several points need to be clarified with regard to the news article by Day. ${ }^{1}$ Most importantly, Italy's new national immunisation plan, issued by a working group of the Italian Ministry of Health, was built on the best available scientific evidence.

The four scientific societies that in 2014 released the second edition of the "lifetime immunization schedule," mentioned in the news article, were consulted by the working group for technical and scientific support. In particular, representatives of the Italian Society of Hygiene, Preventive Medicine and Public Health; the Italian Society of Pediatrics; the Italian Federation of Community Pediatricians; and the Italian Federation of General Practitioners were invited to two formal hearings. Moreover, although the lifetime immunisation schedule constituted a solid basis for discussion during the drafting of the new plan, not all immunisation programmes that it recommended were included in the plan (such as the recommendation to decrease the age threshold for universal free influenza immunisation to age 50 years).

The working group found convincing evidence and pharmacoeconomic data supporting the efficacy, effectiveness, and safety of the newly proposed vaccination programmes. For example:

- Scientific data supported the great potential of human papillomavirus (HPV) immunisation in males to protect against several cancers in both sexes ${ }^{3}$

- Vaccination against rotavirus is offered to all newborns in many European countries whose authorities have considered its optimal cost effectiveness profile ${ }^{4}$

- Pneumococcal conjugate vaccination in at risk categories and in selected age based cohorts of older people is already offered in half of Italian regions on the basis of robust scientific evidence. ${ }^{5}$

Day does not mention that on 15 October 2015 the technical board of the State Regions Conference, whose members represent all Italian regions, released a formal positive position statement on the new national immunisation plan draft issued by the ministry of health. Italian immunisation coverage rates have been decreasing since 2012 and are still below national targets, ${ }^{6}$ with a high degree of heterogeneity across different regions and different vaccines. For example, in Piedmont MMR (measles, mumps, and rubella) vaccine coverage does not reach $90 \%$ for the first dose (the WHO target is $95 \%$ ) and HPV vaccination does not reach the $70 \%$ minimum target coverage for all invited birth cohorts. ${ }^{6}$

A comprehensive framework of immunisation policies in Italy should have mentioned that, because some Italian regions have already adopted the immunisation offer included in the new plan, its adoption at the national level is a powerful tool to harmonise immunisation strategies across the regions. Ultimately it should ensure equity in access to prevention of infectious diseases to all citizens. Currently, there are no differences in the burden of infectious diseases across the Italian regions that could justify different immunisation strategies.

Last but not least, economic aspects deserve a comment. In recent years, Italy has not been able to invest $5 \%$ of total health expenditure in prevention, as had been planned. This missing investment needs to be considered when allocating resources. The estimated increase of the total immunisation bill associated with the new plan ( $€ 200-300 \mathrm{~m}$; $£ 145-217 \mathrm{~m} ; \$ 220-330 \mathrm{~m}$ ) is barely $0.002 \%$ of total Italian health expenditure and $0.04 \%$ of current expenditure for prevention services. In addition, the new plan's budget complements the new immunisation offer 
endorsing and transposing recent EU recommendations in terms of strengthening healthcare providers' training, health education interventions, and immunisation services, as well as implementing immunisation registries at the national level.

Competing interests: During the past two years CS was part of a Sanofi Pasteur MSD advisory board on HPV-9 vaccine and he was invited to five conferences where expenses reimbursements were supported by Pfizer, Sanofi Pasteur MSD, and Reckitt Benkiser. During the past two years PB received grants for joining GSK, Sanofi Pasteur MSD, and Pfizer advisory boards and reimbursements of expenses for participation in conferences.

Full response at: www.bmj.com/content/351/bmj.h6181/rr-4.
1 Day M. Italian expert questions need for expanded vaccination schedule. BMJ 2015;351:h6181. (16 November.)

2 Bonanni P, Azzari C, Castiglia P, et al. [The 2014 lifetime immunization schedule approved by the Italian scientific societies. Italian Society of Hygiene, Preventive Medicine, and Public Health. Italian Society of Pediatrics. Italian Federation of Pediatric Physicians. Italian Federation of General Medical Physicians]. Epidemiol Prev 2014;38:131-46.

3 Audisio RA, Icardi G, Isidori AM, et al. Public health value of universal HPV vaccination Crit Rev Oncol Hematol 2015 Aug 4 [Epub ahead of print]. doi:10.1016/..critrevonc.2015. 07.015.

4 Bonanni P, Signorelli C. Anti-rotavirus: no evidence to discontinue the universal vaccination policy. Ig Sanita Pubb/ 2015;5:559-67.

5 Bonten MJ, Huijts SM, Bolkenbaas M. Vaccine against pneumococcal pneumonia in adults. N Engl J Med 2015;73:93.

6 Bonanni P, Ferro A, Guerra R, et al. Vaccine coverage in Italy and assessment of the 2012-2014 national immunization prevention plan. Epidemiol Prev 2015;39:146-58.

7 Odone A, Fara GM, Giammaco G, et al. The future of immunization policies in Italy and in the European Union: the Declaration of Erice. Hum Vaccin Immunother 2015;11:1268-71.

Cite this as: BMJ 2015;351:h6775

(c) BMJ Publishing Group Ltd 2015 